\title{
Bei schwerer Neurodermitis ins Hochgebirge
}

Neurodermitis wie auch Psoriasis sind häufige Hauterkrankungen, unter denen viele Patienten jahrelang leiden. Bei manchen sind die Symptome so schlimm, dass nur noch ein Aufenthalt in den Bergen oder am Meer Linderung bringt.

Im Juli 2008 war Frau R. am Ende ihrer Kräfte. Der starke Juckreiz trieb die Industriekauffrau aus dem deutschen Ruhrgebiet schier zur Verzweiflung. Den Ärzten bot sich ein massiver Befund: $80 \%$ der Haut waren extrem gerötet, teils angeschwollen, teils stark schuppend und nässend. Überall liessen sich Kratzspuren feststellen. Frau R. konnte die Hände kaum noch bewegen; zudem litt sie unter erheblichen Schlafstörungen. Seit Geburt leidet sie unter einer schweren Neurodermitis. In der Pubertät kam Heuschnupfen dazu. Zudem bestehen Allergien gegen Hausstaubmilbe, Baum- und Gräserpollen sowie Tierhaare.

\section{Weniger Allergene und Schadstoffe}

Bei solch schweren Symptomen einer atopischen Hauterkrankung ist laut Fachleuten ein stationärer Aufenthalt unter den reizklimatischen Bedingungen des Hochgebirges sinnvoll. Entsprechende Therapien bietet die auf 1560 Meter über Meer gelegene Hochgebirgsklinik Davos an. «Das besondere Klima im Hochgebirge bietet vor allem Schadstoffarmut und Allergenarmut», erklärt Claudia Steiner [1], langjährige Co-Chefärztin der Klinik für Dermatologie und Allergologie in Davos. Tatsächlich ist die Pollenbelastung der Luft auf fast 1600 Meter Höhe sehr viel geringer als im Flach- land. Das zeigt ein Messvergleich mit Birkenpollen zwischen Davos und Zürich: Während Davos ein Maximum von 157 pro Kubikmeter erreichte, lag der entsprechende Wert für Zürich mit 3200 pro Kubikmeter fast um das Zwanzigfache höher. Ein weiterer Pluspunkt der Hochgebirgslage ist das besondere Strahlenklima: Die lange Sonnenscheindauer und die intensivere UV-Strahlung (15-20\% mehr UVB) stärken das körpereigene Abwehrsystem. Durch den relativen Sauerstoffmangel wird der Kreislauf

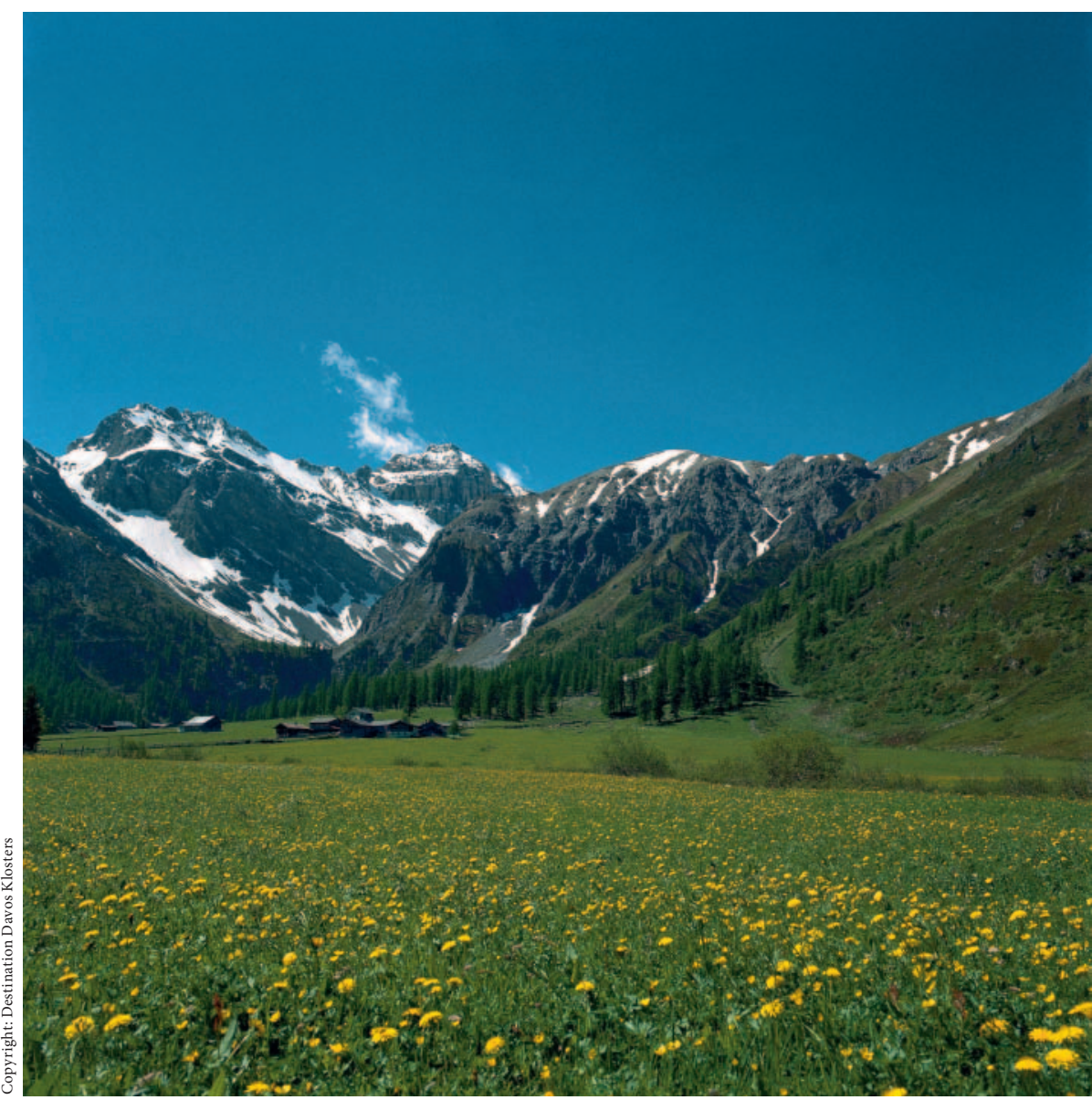

Abb. 1. Nützt bei schweren Hautkrankheiten: das Hochgebirgsklima in Davos.

\section{KARGER}

Fax +49761 4520714 Information@Karger.de www.karger.com 
aktiviert, die Atmung vertieft und damit die Hautdurchblutung verbessert. Des Weiteren produziert der Körper höhenklimatisch bedingt mehr Kortisol. Und: «Die niedrige Luftfeuchtigkeit erhöht die Abdunstung der Haut, mit der Erniedrigung der Hauttemperatur erfolgt eine Minderung des Juckreizes», so Claudia Steiner. Nicht zu unterschätzen seien Ruhe und Entfernung vom Alltag und vom Arbeitsplatz, die sich günstig auf Psyche und Haut auswirkten.

Deshalb können sich Neurodermitis- und Psoriasispatienten, die im Flachland resistent gegen jegliche Therapie geworden sind, in der Höhenlage von ihren Beschwerden weitgehend erholen. So auch Frau R.: Durch Aufenthalte in der Hochgebirgsklinik, so berichtet die hausinterne Patientenzeitschrift, konnte bei ihr immer wieder eine Abheilung und Stabilisierung der Haut erreicht werden. Der gute Hautzustand hielt oft für 1-2 Jahre an, sodass die Patientin nur noch Pflegecremes einsetzen musste. Auch die Heuschnupfenbeschwerden liessen deutlich nach. «Die Therapie der Haut erfolgt mit einem dem Hautzustand angepassten Externum, nach Möglichkeit kortisonfrei, sowie Bädern und einer ergänzenden UV-Therapie», erläutert Claudia Steiner. Bei Muskelverspannungen werden Physiotherapie, Krankengymnastik und Massagen angeboten. Teil des Konzepts sind auch psychologische Beratung bzw. Psychotherapie, denn psychosozialer Stress trägt bei chronischen Dermatosen häufig dazu bei, die Situation zu verschlimmern.
Dass die Hochgebirgsklimatherapie tatsächlich wirkt, ist mittlerweile auch wissenschaftlich belegt, wie eine in der Deutschen Medizinischen Wochenschrift publizierte Übersichtsarbeit [2] kürzlich gezeigt hat. Professor Angela Schuh von der LudwigMaximilians-Universität München und ihr Kollege Dennis Nowak haben hierzu die medizinische Literatur der letzten 15 Jahre durchsucht und sind dabei auf Studien gestossen, die zwar nicht alle, aber viele Behauptungen belegen. Wissenschaftlich bewiesen ist danach, dass die Hochgebirgsklimatherapie atopische Krankheiten bessern kann - neben Neurodermitis auch Heuschnupfen und das allergische Asthma. Die Mehrzahl der Patienten hat nach einem mehrwöchigen Aufenthalt im Hochgebirge keine Symptome mehr; bei Erwachsenen verschaffen auch Mittelgebirgshöhen eine Linderung. Professor Schuh: «Der Klimastress fördert die körpereigene Bildung von Kortison. Die Wirkung hält oft nach der Rückkehr an.» In einer Studie verzeichneten zwei Drittel der Patienten noch nach 12 Monaten eine deutliche Verbesserung, berichten Schuh und Nowak. Auch Menschen mit nicht allergischem Asthma bekommt die schadstoffarme Hochgebirgsluft. Umstritten sei allerdings, ob die hohe Ozonkonzentration im Hochgebirge ein Nachteil ist.

Die intensivere UV-Strahlung fördert bei Menschen mit Psoriasis die Abheilung der Schuppenflechte; bei älteren Menschen mit Osteoporose wurde ein Anstieg des Knochenvitamins D3 gemessen. Menschen mit Bluthochdruck profitieren von den Kältereizen und dem leichten Sauerstoffmangel.
Eine andere Form der Klimatherapie ist der Aufenthalt am Meer, wo die hohe Luftfeuchtigkeit und der Salzgehalt der Luft eine zusätzliche schleimlösende Wirkung auf die Atemwege haben. Zur Wirkung dieser Badetherapien an Nord- und Ostsee haben Schuh und Nowak nur wenige aktuelle Studien gefunden. Diese zeigen jedoch, dass das Reizklima bei Kindern und Erwachsenen eine Neurodermitis bessert. Die Meeresluft steigert auch die Bildung von Antikörpern und körpereigenem Kortison. Die positive Wirkung der Mittelmeer- und Atlantikluft ist vor allem durch Studien aus Skandinavien belegt, von wo aus viele Patienten mit Neurodermitis und Psoriasis anreisen.

Besonders günstige Bedingungen herrschen am Toten Meer: Die intensive UV-Strahlung und das Meerwasser mit seinem extrem hohen Salzgehalt verschaffen vor allem Patienten mit Neurodermitis und Psoriasis eine rasche und nachhaltige Linderung. Professor Schuh: «Bei fast allen Patienten bessert sich der Hautzustand, was bei Neurodermitikern über mehrere Monate, bei Psoriatikern sogar über ein Jahr anhalten kann.» $(r f i)$

\section{Literatur}

1 Steiner C: Neurodermitis und Psoriasis: Was bringt die stationäre Therapie im Hochgebirgsklima mehr? Praxis 2009;98:13731376.

2 Schuh A, Nowak D: Klimatherapie im Hochgebirge und im Meeresklima. Evidente Akut- und Langzeiteffekte - ein qualitativer Review. DMW Dtsch Med Wochenschr 2011; 136:135-139. 